\title{
Ceratite dendritiforme em usuários de lentes de contato
}

\author{
Caro editor,
}

Temos acompanhado um aumento importante no número de casos de ceratite infecciosa causada por Acanthamoeba no ambulatório de Doenças Externas e Córnea da Universidade Federal de São Paulo. Até o início da década passada este protozoário era uma causa incomum de ceratite. Entretanto, através da análise dos dados do nosso laboratório, observamos que houve um aumento importante do número de pacientes acometidos por esta infecção(1).

Devido às dificuldades no manejo clínico destes pacientes e ao freqüente comprometimento da visão ${ }^{(2)}$, consideramos que as medidas de prevenção são a melhor abordagem para limitar o impacto desta doença na nossa sociedade. Os usuários de lente de contato devem ser bem orientados quanto aos cuidados com as suas lentes (e.g. não usar salina caseira, abolir o uso de soro fisiológico como solução única na manutenção das lentes), e até mesmo desestimulados a usá-las quando detectamos falhas na higiene das lentes.

Após a instalação da infecção, o tratamento é normalmente demorado e de alto custo $^{(3)}$. A ceratite por Acanthamoeba apresenta duas formas distintas e a eficácia do tratamento depende da forma clínica na qual se detectou a infecção. A forma epitelial é a mais precoce. Nesta fase o parasita está limitado ao epitélio e há maior probabilidade de recuperação. Posteriormente, há invasão do estroma da córnea (forma estromal), com intensa inflamação e necrose. Nesta fase tardia, a eficácia do tratamento é significativamente menor ${ }^{(4)}$.

O maior empecilho ao tratamento na fase epitelial é a demora no diagnóstico. Durante esta fase a infecção pode não ser diagnosticada pois apresenta características inespecíficas como irregularidade epitelial, ceratite ponteada e defeito epitelial. Além destes achados, uma forma semelhante à ceratite dendritiforme, leva freqüentemente ao diagnóstico equivoca- do de ceratite herpética ${ }^{(3)}$. Nos nossos pacientes este tem sido o fator mais importante de diagnóstico tardio.

Como sugestão, recomendamos uma abordagem específica em pacientes usuários de lente de contato com diagnóstico de ceratite dendritiforme, especialmente naqueles nunca antes diagnosticados com ceratite herpética. Nestes pacientes, consideramos que ainda que o oftalmologista considere ceratite herpética como primeiro diagnóstico, deva ser feito o debridamento da lesão, recomendado tanto para ceratite herpética quanto para ceratite por Acanthamoeba ${ }^{(3-6)}$. O material recolhido deve ser enviado para análise laboratorial, quando disponível, que inclua análise direta e cultura em meio especial para Acanthamoeba.

Apesar do aumento do custo do manejo destes pacientes, consideramos que o grave acometimento visual em pacientes usualmente em idade produtiva que a infecção por Acanthamoeba pode causar justifica o cuidado adicional. A análise do custo/benefício da abordagem que propomos, entretanto, somente seria possível com o cálculo exato da freqüência e etiologia da ceratite dendritiforme em usuários de lentes de contato na nossa população.

\section{REFERENCIAS}

1. Hofling-Lima AL. Infecções oculares exógenas por bactérias, fungos e parasitas. [monografia em CD-ROM]. São Paulo; 2002.

2. Freitas D. Infecções emergentes em oftalmologia; ceratite por Acanthamoeba [tese]. São Paulo: Universidade Federal de São Paulo; 2000

3. Alvarenga L, Freitas D, Hofling-Lima AL. Ceratite por Acanthamoeba. Arq Bras Oftalmol 2000;63:155-60.

4. Kumar R, Lloyid D. Recent advances in the treatment of Acanthamoeba keratitis. Clin Infect Dis 2002;35:434-41.

5. Sharma S. Keratitis. Biosci Rep 2001;21:419-44.

6. Freitas D, Alvarenga L, Hofling-Lima AL. Ceratite herpética. Arq Bras Oftalmol 2001;64:81-6.

' Doutor em Oftalmologia, Setor de Doenças Externas Oculares e Córnea, Departamento de Oftalmologia - Universidade Federal de São Paulo - UNIFESP.

${ }^{2}$ Livre-Docente, Professora do curso de Pós-Graduação em Oftalmologia, Departamento de Oftalmologia - Universidade Federal de São Paulo - UNIFESP.

Endereço para correspondência: Denise de Freitas. Universidade Federal de São Paulo - (UNIFESP) - Depto. de Oftalmologia

Rua Botucatu, 822 - São Paulo - SP. E-mail: dfreitas@pobox.com 